\title{
Intracellular Evaluation of ER Targeting Elucidates a Mild Form of Inherited Coagulation Deficiency
}

\author{
Lara Rizzotto, ${ }_{1}^{1}$ Mirko Pinotti, ${ }^{1}$ Paolo Pinton, ${ }^{2}$ Rosario Rizzuto, ${ }^{2}$ and Francesco Bernardi ${ }^{1}$ \\ ${ }^{1}$ Department of Biochemistry and Molecular Biology, University of Ferrara, Via Fossato di Mortara 74, Ferrara, Italy; \\ ${ }^{2}$ Department of Experimental and Diagnostic Medicine, Section of General Pathology, Telethon Center for Cell Imaging and Interdis- \\ ciplinary Center for the Study of Inflammation, University of Ferrara, Italy
}

\begin{abstract}
Missense mutations reduce protein levels through several molecular mechanisms. Among them, altered targeting to endoplasmic reticulum (ER) and its relationship with clinical phenotypes in patients have been poorly investigated. To address this point, we studied the prepeptide mutations (L-48P, L-42P) associated with mild deficiency of factor VII (FVII), the serine-protease triggering blood coagulation. Mutations were introduced into the native FVII to evaluate secreted and intracellular protein levels, and into a chimeric FVII-GFP to study ER targeting in living cells. In conditioned medium from stably or transiently transfected cells, expression levels of the $-48 \mathrm{PFVII}$ ( $9 \%$ and $55 \%$, respectively) and particularly those of the $-42 \mathrm{PFVII}(2 \%$ and $12 \%$ ) were decreased compared with those of WtFVII, indicating the causative nature of mutations. Markedly reduced protein levels were observed in cell organelles for -48PFVII (10.5 $\pm 4.9 \mathrm{ng} / \mathrm{mL}$; Wt-FVII, $130 \pm 43.4 \mathrm{ng} / \mathrm{mL})$ and $-42 \mathrm{PFVII}(\sim 5 \mathrm{ng} / \mathrm{mL})$, thus suggesting impaired ER targeting. Fluorescence of the -48PFVII-GFP and -42PFVII-GFP was diffuse, covered the nucleus, and declined upon plasma membrane permeabilization with digitonin, which demonstrated mislocalization of variants in the cytosol. Noticeably, the residual fluorescence of -48PFVII-GFP (10\%) and -42PFVII-GFP (20\%) in organelles was fairly compatible with FVII levels in patients' plasma. The studies with the native and chimeric proteins indicated that both prepeptide mutations were associated with residual expression of normal FVII, which explained the mild form of FVII deficiency in patients. This approach, extendable to other coagulation serine proteases, clearly contributed to elucidate the relationship of genotype with plasma and clinical phenotype.

Online address: http://www.molmed.org
\end{abstract}

doi: 10.2119/2005-00040.Rizzotto

\section{INTRODUCTION}

Blood coagulation involves numerous serine-proteases with procoagulant and anticoagulant function $(1,2)$, whose plasma levels are regulated to maintain the hemostatic balance and prevent hemorrhagic or thrombotic events.

Partial deficiency of procoagulant factors is associated with variable hemorrhagic phenotypes $(3,4)$, ranging from asymptomatic to very severe. These observations, together with the notion that levels above $10 \%$ of normal plasma are sufficient to prevent major hemorrhagic symptoms, make the characterization of residual levels of great importance to understand and classify coagulation deficiencies.

The extensive genetic investigations in patients revealed the mutational pattern for a number of inherited hemorrhagic coagulation factor disorders (5-10), which is mainly characterized by missense mutations reducing circulating protein levels in plasma. So far, the molecular mechanisms through which these mutations impair the intracellular biosynthetic pathways, and their relationship with plasma and clinical phenotype in patients, have been poorly investigated (11-16). Missense mutations affecting the prepeptide region of coagulation factors (5-10), crucial for targeting of nascent molecules to the endoplasmic reticulum (ER) (17), represent peculiar models to address this issue. The causative role of substitutions affecting this region, and their effects on protein levels, are difficult to predict because of the extreme variability of prepeptide sequences $(18,19)$ even among members of the coagulation serine protease family.
Address correspondence and reprint requests to Mirko Pinotti, University of Ferrara, Department of Biochemistry and Molecular Biology, Via Fossato di Mortara 74, 44100 Ferrara, Italy. Phone: +39 0532 424424; fax: +39 0532 424484; e-mail: pnm

Submitted November 25, 2005; accepted for publication July 22, 2006.
In the present study, through in vitro expression of native as well as of chimeric proteins with green fluorescent protein (GFP) (20), we elucidated the effect within living cells of the prepeptide mutations in factor VII (FVII), the serine protease triggering the clotting cascade $(21,22)$.

\section{MATERIALS AND METHODS}

\section{FVII Mutations}

The mutations in the FVII prepeptide have been selected from the FVII mutation database (8) and the International Registry of FVII deficiency (9). The homozygous L-48P mutation was associated with FVII activity (FVIIc) and antigen (FVIIag) levels of $11 \%$ (23). The L-42P substitution, in doubly heterozygous condition with the V252M change, was associated with FVIIc levels of $12 \%$ (24).

\section{Expression Vectors, Cell Culture, and Transfection}

To make the pWtFVII-GFP vector, the FVII coding sequence cloned in the 
pCDNA3-FVII (25) was amplified with PfuTurbo DNA polymerase (Stratagene, La Jolla, CA, USA) using the forward primer in the CMV promoter (5'-GGC GTGTACGGTGGGAGGTC-3') and the reverse primer designed to abrogate the FVII stop codon and to introduce a BamH1 site (5'-GTGAATGGATCCGGG AAATGGGCTCGCAG-3'). The amplified fragment was digested with BamH1 and cloned in frame with the coding sequence of the enhanced GFP, inserted in the pCDNA3 vector (26).

Mutations were introduced into the pCDNA3-FVII and pWtFVII-GFP vectors using the Quick-Change Mutagenesis Kit (Stratagene) with the following oligonucleotides: 5'-CTCTGCCTTCTGCCTGGG CTTCAGGGC-3'(L-48P), 5'-GGGCTTCAG GGCTGCCCGGC TGCAGTCTTCGTA ACCC-3' (L-42P) and 5'-CGTGGTACC TGACGGTCATCGTCAGCT GG-3' (G360V). The plasmid expressing the cytosolic protein kinase $\mathrm{C} \beta$-GFP (pPKC $\beta$ GFP) was available in our laboratory (26).

BHK cells, known to correctly process FVII, were transfected as previously reported (25). For fluorescence microscopy, cells were cultured on 24-mm glass coverslips.

Stable cell lines were established as previously described (27). Among 24 stable clones for each variant, the 3 highest producers were selected by ELISA and studied.

\section{FVII Expression Levels}

FVII antigen was measured by ELISA (Asserachrom, Diagnostica Stago). As previously described (28), the activity of recombinant FVII was evaluated by its ability to activate its physiologic substrate factor (F) X (FXa generation assays) and expressed as relative fluorescence units (Rfu) s${ }^{-1} \mathrm{nM} \mathrm{FVII}{ }^{-1}$. Medium from cells transfected with the gutted pCDNA3 was used as negative control.

\section{Fluorescence Microscopy}

Cells were observed $48 \mathrm{~h}$ posttransfection using a Zeiss-Axiovert 200M fluorescence microscope (470 $\mathrm{nm}$ excitation, $505 \mathrm{~nm}$ emission). After digitonin treat- ment, fluorescence was monitored at 5-s intervals for $5 \mathrm{~min}$.

Images were analyzed using the Metamorph/Metafluor 4.5 software.

\section{Digitonin Treatment}

Transfected cells were treated with $200 \mu \mathrm{M}$ digitonin in medium mimicking intracellular ion composition $(140 \mathrm{mM}$ $\mathrm{KCl}, 10 \mathrm{mM} \mathrm{NaCl}, 1 \mathrm{mM} \mathrm{K} \mathrm{PO}_{4}, 5.5 \mathrm{mM}$

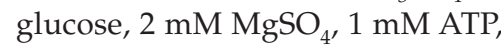
$2 \mathrm{mM}$ sodium succinate, $20 \mathrm{mM}$ Hepes, $\mathrm{pH}$ 7.05). Digitonin complexes specifically with unesterified 3- $\beta$-hydroxysterols, forming in situ precipitates and selectively perforating the plasma membrane, leaving ER and Golgi apparatus membranes sealed.

\section{Subcellular fractionation}

Cells were washed twice with $1 \times$ icecold PBS and harvested via scraping into $1 \mathrm{~mL}$ CLB (10 mM HEPES, $10 \mathrm{mM} \mathrm{NaCl}$, $1 \mathrm{mM} \mathrm{KH} \mathrm{PO}_{4}, 5 \mathrm{mM} \mathrm{NaHCO}{ }_{3}, 1 \mathrm{mM}$ $\mathrm{CaCl}_{2}, 0.5 \mathrm{mM} \mathrm{MgCl}$ )/ 5 mM EDTA/ $1 \mathrm{mM}$ PMSF. The homogenate was centrifuged at $110.000 \mathrm{~g}$ in a TLA 100.4 rotor (Beckman, Fullerton, CA) for $30 \mathrm{~min}$. The resulting supernatant, containing the soluble plasma (cytosolic) proteins, was conserved for further assays. The pellet, containing nuclei, debris, and membrane proteins, was resuspended in $1 \mathrm{~mL}$ lysis buffer (50 mM Tris, pH 7.4, $150 \mathrm{mM}$ $\mathrm{NaCl}, 1 \%$ Triton $\mathrm{X}-100,1 \mathrm{mM}$ PMSF), incubated on ice for $30 \mathrm{~min}$, and centrifuged at $10.000 \mathrm{~g}$ at $4{ }^{\circ} \mathrm{C}$ for $10 \mathrm{~min}$ to clear cellular debris from the lysate. The supernatant was removed and conserved for further assays.

Putative cytosolic and organelle fractions were run on SDS-PAGE (NuPAGE 4-12\% bis-tris gel; Invitrogen, Carlsbad, CA, USA) under reducing conditions. Membrane was probed with monoclonal antibodies directed against the $\beta$-actin (Cell signaling Technology, Danvers, MA, USA) or the protein disulphide isomerase (Calbiochem, San Diego, CA, USA). After incubation with the primary antibody, filters were incubated with the secondary anti-rabbit antibody (Pierce, Rockford, IL, USA).

\section{Computational Analysis}

The amino acid sequence of FVII prepeptide was analyzed for secondary structure prediction with SOPMA (http:/ / npsa-pbil.ibcp.fr/cgi-bin/npsa_automat. pl?page = /NPSA/npsa_sopma.html) and for the signal peptide probability score by exploiting the following computational tools: SignalP 3.0 (http:/ / www. cbs.dtu.dk/services/SignalP/), PSORT II (http://psort.nibb.ac.jp), TargetP 1.1 (http://www.cbs.dtu.dk/services/ TargetP), SigFind (http://139.91.72.10/ sigfind/ sigfind.html), PrediSi (http:/ / www.predisi.de), and Predotar (http:/ / genoplante-info. infobiogen.fr/predotar/).

\section{RESULTS}

\section{Computational Analysis}

Secondary structure analysis (SOPMA method) predicted that the introduction of proline for leucine at positions -48 and -42 in the FVII prepeptide alters and reduces the $\alpha$-helix content of the hydrophobic core (Figure 1A), crucial for proper interaction with the signal recognition particle (17).

When a panel of computational programs devoted to the signal peptide prediction was used, no major differences in the score among prepeptide sequences were observed (Figure 1B).

\section{Expression of Mutations in the Native FVII}

The causative role of the prepeptide mutations was approached through the expression of FVII variants in eukaryotic cells and investigation at both the extracellular and intracellular level. As control, beside the WtFVII, we also expressed the naturally occurring 360VFVII variant that possesses a correct prepeptide sequence and a Gly-to-Val substitution in the C-terminal domain.

\section{Expression Levels in Conditioned Medium}

Expression levels of FVII variants in medium were evaluated in 5 independent transient transfection experiments. Mean FVII protein levels of WtFVII in 
A)

Factor VII prepeptide

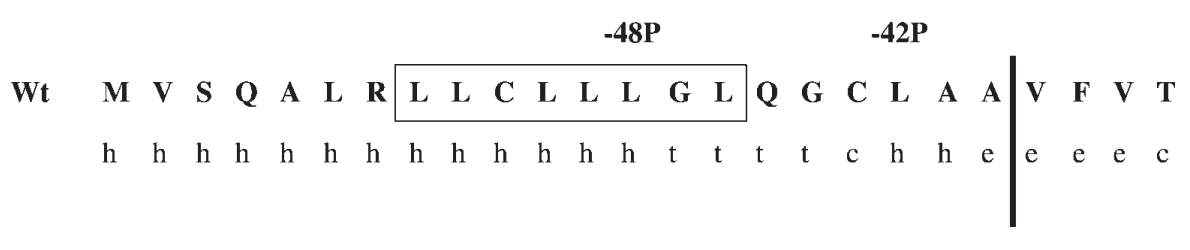

B)

\begin{tabular}{|c|c|c|c|}
\hline Prediction Method & Wt-FVII & -48 PFVII & -42 PFVII \\
\hline SignalP & 0.99 & 0.99 & 0.99 \\
\hline PSORTII & 5.95 & 5.08 & 5.95 \\
\hline Targetp & 0.917 & 0.873 & 0.901 \\
\hline PredISi & 1.0000 & 0.9664 & 0.9874 \\
\hline SigFind & 0.9885 & 0.9764 & 0.9797 \\
\hline Predotar & 0.99 & 0.96 & 0.99 \\
\hline
\end{tabular}

Figure 1. Computational analysis of FVII prepeptide sequence. (A) Amino acid sequence of the FVII prepeptide and prediction of the secondary structure. Amino acid substitutions are indicated. The hydrophobic core is shown (box). The vertical bold line represents the signal peptidase cleavage site. $h$, helix; $c$, random coil; e, extended strand; $t, \beta$ turn. (B) Prediction of the signal peptide score with different computational programs.

medium at the steady state $(24-48 \mathrm{~h}$ posttransfection) were $125 \pm 32 \mathrm{ng} / \mathrm{mL}$, whereas levels of the 360VFVII were undistinguishable from those of the negative control. Protein levels of the $-42 \mathrm{PFVII}$ and $-48 \mathrm{PFVII}$ variants were $15.4 \pm 5.7 \mathrm{ng} / \mathrm{mL}$ ( $12 \%$ of WtFVII) and $69.1 \pm 20.1 \mathrm{ng} / \mathrm{mL}$ ( $55 \%$ of WtFVII), respectively (Figure 2B).

The activity of FVII was investigated in these media through fluorogenic FXa generation assays, enabling us to detect even very low levels. FVII activity of the 360V-FVII was undetectable, whereas that of the $-42 \mathrm{PFVII}$ and $-48 \mathrm{PFVII}$ variants was $11 \% \pm 5 \%$ and $66 \% \pm 3 \%$ of WtFVII, respectively (Figure 2A).

The expression levels of the prepeptide variants were also measured at 6 and 12 $h$ posttransfection through functional assays. Although expression levels of the -42 PFVII were barely detectable, those of the $-48 \mathrm{PFVII}$ at 6 and $12 \mathrm{~h}$ posttransfection were $15 \% \pm 3 \%$ and $53 \% \pm 12 \%$ of WtFVII, respectively (Figure 2A).
Cells stably expressing WtFVII and prepeptide variants were also selected and investigated. The secreted protein levels of -42 PFVII $(30 \pm 10 \mathrm{ng} / \mathrm{mL})$ and -48 PFVII $(117 \pm 39 \mathrm{ng} / \mathrm{mL})$ from the 3 best clones were significantly lower than those of WtFVII (1314 $\pm 490 \mathrm{ng} / \mathrm{mL}$ ) (Figure $2 \mathrm{C}$ ). These concentrations permitted us to evaluate the specific activity of the -42PFVII (2092 $\pm 117 \mathrm{Rfu} / \mathrm{sec} / \mathrm{nMFVII})$ and $-48 \mathrm{PFVII}(2125 \pm 493 \mathrm{Rfu} / \mathrm{sec} / \mathrm{nMFVII})$ molecules, which was comparable to that of WtFVII (2100 $\pm 553 \mathrm{Rfu} / \mathrm{sec} / \mathrm{nMFVII})$.

\section{Intracellular FVII Expression Levels}

To evaluate FVII proteins at the intracellular level, lysates of either transiently or stably transfected cells were processed to separate cytosol from organelles. The successful fractionation of cell content was indicated by Western blotting analysis, which identified $\beta$-actin almost exclusively in the cytosolic fraction and the protein disulphide isomerase (PDI) almost exclusively in the organelle fraction (Figure 2B-C, right panels).

FVII protein levels inside cell organelles of transiently transfected cells were detectable only for the WtFVII $(10.3 \pm 3.7 \mathrm{ng} / \mathrm{mL})$ and the $360 \mathrm{VFVII}$ $(22.8 \pm 4.4 \mathrm{ng} / \mathrm{mL})$ but not for the $-42 \mathrm{PFVII}$ and $-48 \mathrm{PFVII}$ variants (Figure 2B, left panel). In contrast, investigations in stable clones revealed the presence of very reduced protein levels in organelles for the -48 PFVII $(10.5 \pm 4.9 \mathrm{ng} / \mathrm{mL})$ compared with those of Wt-FVII (130 \pm $43.4 \mathrm{ng} / \mathrm{mL}$ ) (Figure 2C). The $-42 \mathrm{PFVII}$ levels in this compartment ranged around the sensitivity limit of the ELISA $(\sim 4 \mathrm{ng} / \mathrm{mL})$, and reached values of 4.1 and $6.1 \mathrm{ng} / \mathrm{mL}$ in 2 clones.

FVII protein levels in the cytosolic fractions from transiently or stably transfected cells were not measurable by ELISA. This is likely explained by the inability of the anti-human FVII antibody to recognize the improperly folded FVII molecules in the cytosol environment, which does not permit the required extensive posttranslational modifications of FVII to occur (29).

\section{Expression of Mutations in the FVII- GFP Chimera}

To investigate at the intracellular level in living cells the effect of these substitutions on the first biosynthetic steps, FVII was expressed as a fusion protein with the GFP (Figure 3A). Fluorescence of the FVII-GFP variants was evaluated by microscopy of transfected cells.

Cells expressing the -42 PFVII-GFP and the -48PFVII-GFP showed a diffuse fluorescence, also present in the nuclear region (Figure 3D and E, time 0 ). Transfected cells were subsequently treated with digitonin to permeabilize the plasma membrane. In these conditions, the cytosolic cell content leaks out of the permeabilized membranes, leaving ER and Golgi apparatus membranes sealed. Upon digitonin treatment, fluorescence of the -42PFVII-GFP and -48PFVII-GFP rapidly decreased over time (see Figure 3D and E). The residual fluorescence observed for the 


\section{A)}

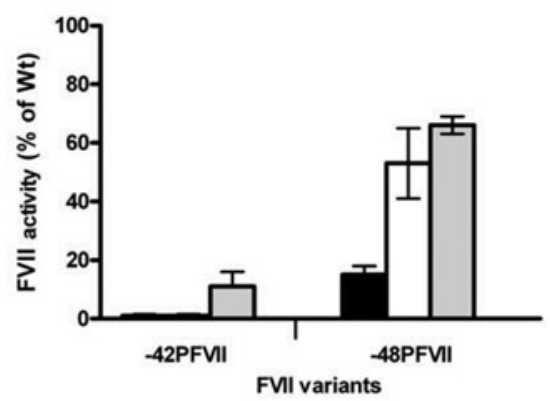

B)

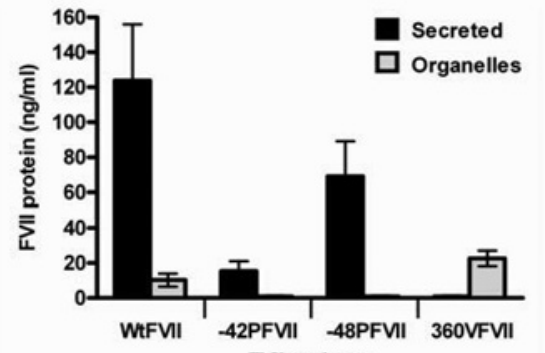

FVI variants

C)

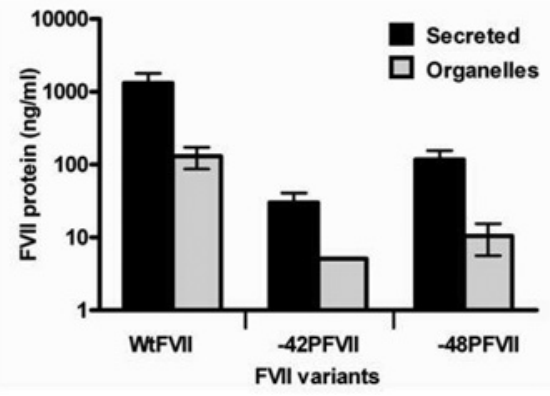

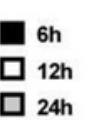
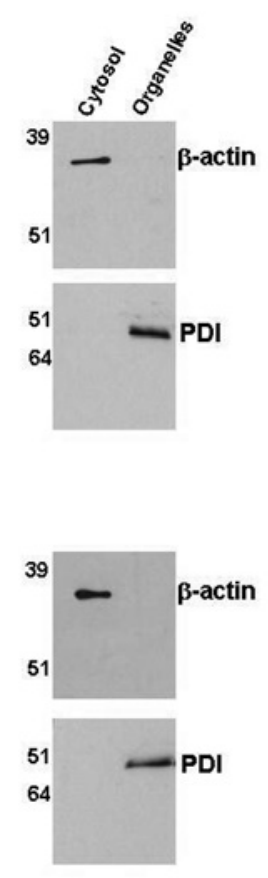

Figure 2. Expression levels of FVII variants. (A) Activity of FVII variants (expressed as $\%$ of WtFVII) in medium collected at 6, 12, and $24 \mathrm{~h}$ after transient transfection (5 independent experiments). (B-C) Left panels. Protein levels of FVII variants in medium and organelles from transiently ( $B, 5$ independent experiments) or stably ( $C, 3$ independent clones) transfected cells in $24 \mathrm{~h}$. Protein levels in $\mathrm{C}$ are reported in log scale to better detail low levels of variants. Results are reported as average \pm standard deviation. (B-C) Right panel. Detection by Western blotting of the $\beta$-actin $(\sim 42 \mathrm{kDa})$ and PDI $(\sim 55 \mathrm{kDa})$ proteins as markers of the cytosolic and organelle fractions. Molecular weights are given in kDa.

-48PFVII-GFP was approximately $10 \%$, while that of the -42PFVII-GFP was approximately 20\% (Figure 3D and E). Similarly, a diffuse fluorescence was observed by expressing the protein kinase $\mathrm{C} \beta$ (PKC $\beta$ ) isoform-GFP chimera, which has been demonstrated to be largely cytosolic (26). After digitonin treatment, the residual fluorescence levels of PKC $\beta$-GFP were indistinguishable from the background (Figure $3 \mathrm{C}$ ). At variance, fluorescence of the WtFVIIGFP was excluded from the nucleus and was maintained over time upon digitonin treatment $(90 \%$ at 180 s; Figure $3 \mathrm{~A}$ ), thus indicating correct targeting of this chimera to ER.

Expression levels of the WtFVII-GFP were also investigated in conditioned medium 48 to $72 \mathrm{~h}$ posttransfection. The ELISA assay, exploiting a capture monoclonal antibody recognizing the aminoterminal portion of FVII and a detecting polyclonal anti-FVII antibody, revealed that levels of extracellular WtFVII-GFP chimera were very low $(2 \pm 1 \mathrm{ng} / \mathrm{mL})$ compared with those of the native FVII (125 $\pm 32 \mathrm{ng} / \mathrm{mL})$. Moreover, the FXa generation activity of the WtFVII-GFP in medium was virtually undetectable. These findings are likely explained by the presence of the GFP attached to the carboxy terminus of the catalytic domain of FVII that is important for secretion (30).

\section{DISCUSSION}

The natural L-48P and L-42P mutations in FVII were chosen as models to elucidate mechanisms of altered biosynthesis and reduction of protein levels and their correlation with plasma and clinical phenotype in patients. Because of their localization at crucial positions of the prepeptide (17), the L-48P (hydrophobic core) and L-42P (position -3 preceding the signal-peptidase cleavage site) changes are candidates to affect targeting of nascent FVII to ER. However, computational analyses failed to predict significant alterations of the prepeptide properties.

Prepeptide variants of the native FVII were initially expressed in transient transfection experiments to evaluate consequences of mutations on secreted and intracellular FVII levels.

The steady-state expression levels in medium of the $-42 \mathrm{PFVII}$ variant were significantly reduced whereas those of the -48 PFVII, though showing an altered early secretion profile, were slightly reduced compared with those of WtFVII. The G360V substitution in the FVII carboxyl-terminal domain, associated to undetectable FVII protein levels in plasma, was investigated as an example of mutation affecting a highly conserved residue in all serine proteases (31) and therefore expected to alter FVII folding/biosynthesis but not targeting to ER. Not surprisingly, the 360VFVII variant was not secreted at appreciable levels in conditioned medium.

Studies at the intracellular level revealed appreciable protein amount of the WtFVII and 360VFVII in organelles, thus indicating access of FVII molecules to the secretory pathway. The significantly 
higher protein levels of the 360VFVII compared with those of WtFVII indicated retention and accumulation of this variant into the organelle lumen. Conversely, the-42PFVII and -48PFVII into organelles were undetectable, which prompted us to select and investigate stably expressing cells.

Comparison among the best expressing clones revealed markedly reduced secretion levels for the $-42 \mathrm{PFVII}$ and -48 PFVII variants, thus clearly indicating the causative role of the L-42P and L-48P changes. On the other hand, both variants possessed a specific activity comparable to WtFVII, which supported correct processing of the $\mathrm{N}$-terminus of the secreted FVII molecules. Investigation of stable clones also unraveled markedly reduced FVII levels of both variants into cell organelles, a finding compatible with impaired targeting of nascent FVII to ER, a first step of the secretory pathway.

The FVII-GFP chimera, which permits direct investigation of protein trafficking in living cells, was exploited to overcome the inability of the ELISA to reveal protein levels into the cytosol. Some limitations of this approach, i.e very low levels of the secreted chimeric proteins, do not preclude an informative analysis of ER targeting. The diffuse fluorescence of the -42PFVII-GFP and -48PFVII-GFP rapidly decreased over time upon treatment of cells with digitonin, thus convincingly demonstrating that these variants were mainly cytosolic and thus excluded from cell organelles. A well-characterized cytosolic protein, used as control, did not produce appreciable fluorescence after plasma membrane permeabilization, thus further indicating that the residual values of chimeric FVII prepeptide variants were attributable to molecules entering the ER lumen. In particular, the residual fluorescence observed in organelles for the -48PFVII-GFP and -42 PFVII-GFP indicated that $10 \%$ to $20 \%$ of mutant proteins entered the ER, a finding compatible with plasma FVII levels of patients ( $11 \%$ to $12 \%$ ).

Taken together, these data suggest the FVII-GFP strategy as an informative tool

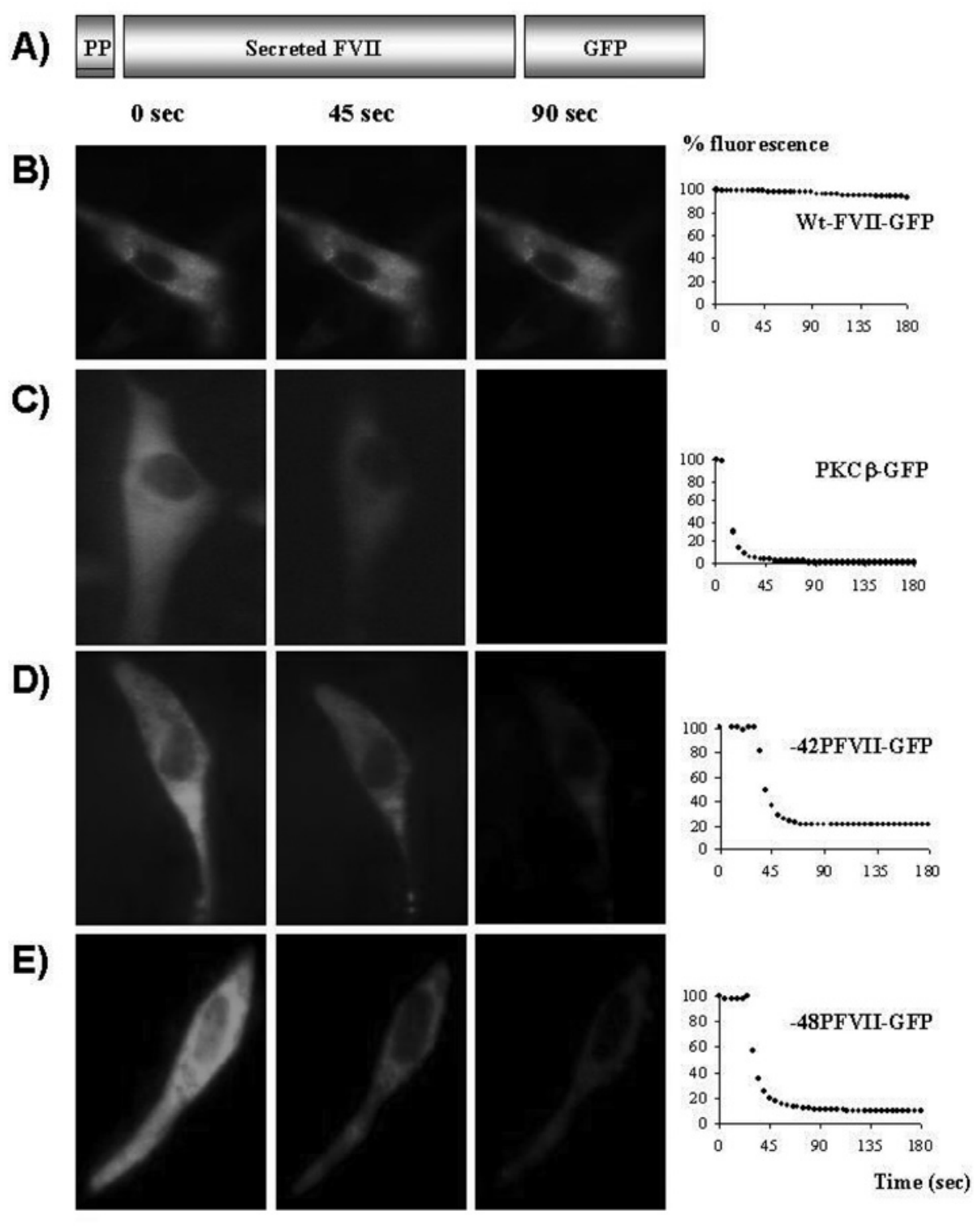

Figure 3. Expression cassette of the FVII-GFP and localization of chimeras within living BHK cells. (A) Schematic representation of the FVII-GFP cassette. PP, FVII preproleader sequence; GFP, enhanced green fluorescence protein. (B-E): Fluorescence images of cells expressing Wt-FVII-GFP (B), PKC $\beta$-GFP (C), -42PFVIIGFP (D), and -48PFVII-GFP (E) before (time 0 ) and 45 or $90 \mathrm{~s}$ after treatment with digitonin. For each construct, the cell shown is representative of at least 50 cells examined in 3 independent experiments. Graphics report the average cell fluorescence over time given as percentage of the fluorescence at time 0 .

to evaluate the effect of FVII prepeptide mutations on ER targeting in living cells. The expression studies with the native and chimeric FVII molecules indicated that both prepeptide mutations were associated to residual FVII levels, which are sufficient to prevent major hemor- rhagic symptoms and to explain the presence in patients of a mild form of FVII deficiency. This approach, extendable to other coagulation serine proteases, clearly contributed to elucidate the relationship of genotype with plasma and clinical phenotype. 


\section{ACKNOWLEDGMENTS}

F.B., M.P., and L.R were supported by Telethon grant GGP05214 and the Italian University Ministry (MURST). R.R. and P.P. were supported by Telethon grant GGP05284, the Italian Association for Cancer Research (AIRC), the Italian University Ministry (MIUR and FIRB), the EU (fondi strutturali Obiettivo 2), the PRRIITT program of the Emilia Romagna Region, and the Italian Space Agency (ASI).

\section{REFERENCES}

1. Dahlback B. (2000) Blood coagulation. Lancet 355:1627-32

2. Mann KG, Butenas S, Brummel K. (2003) The dynamics of thrombin formation. Arterioscler. Thromb. Vasc. Biol. 23:17-25.

3. Mannucci PM, Tuddenham EG. (2001) The hemophilias: from royal genes to gene therapy. N. Engl. J. Med. 344:1773-9.

4. Mannucci PM, Duga S, Peyvandi F. (2004) Recessively inherited coagulation disorders. Blood 104:1243-52.

5. Kemball-Cook G, Tuddenham EG, Wacey AI. (1998) The factor VIII Structure and Mutation Resource Site: HAMSTeRS version 4. Nucleic Acids Res. 26:216-9.

6. Giannelli F et al. (1998) Haemophilia B: database of point mutations and short additions and deletions, eighth edition. Nucleic Acids Res. 26:265-8.

7. Millar DS et al. (2000) Molecular analysis of the genotype-phenotype relationship in factor X deficiency. Hum Genet. 6:249-57.

8. McVey JH, Boswell E, Mumford AD, KemballCook G, Tuddenham EG. (2001) Factor VII deficiency and the FVII mutation database. Hum. Mutat. 17:3-17.

9. Mariani G et al. (2005) Clinical phenotypes and factor VII genotype in congenital factor VII deficiency. Thromb. Haemost. 93:481-7.

10. Saunders RE, O'Connell NM, Lee CA, Perry DJ, Perkins SJ. (2005) Factor XI deficiency database: an interactive web database of mutations, phenotypes, and structural analysis tools. Hum. Mutat. 26:192-8.

11. Racchi M, Watzke HH, High KA, Lively MO. (1993) Human coagulation factor X deficiency caused by a mutant signal peptide that blocks cleavage by signal peptidase but not targeting and translocation to the endoplasmic reticulum. J. Biol. Chem. 268:5735-40.

12. Wang WB et al. (2005) Factor X Shanghai and disruption of translocation to the endoplasmic reticulum. Haematologica 90:1659-64.

13. Fitches AC, Appleby R, Lane DA, De Stefano V, Leone G, Olds RJ. (1998) Impaired cotranslational processing as a mechanism for type I antithrombin deficiency. Blood 92:4671-6.
14. Hunault M, Arbini AA, Carew JA, Peyvandi F, Bauer KA. (1999) Characterization of two naturally occurring mutations in the second epidermal growth factor-like domain of factor VII. Blood. 93:1237-44.

15. Yamazaki T, Nicolaes GA, Sorensen KW, Dahlback B. (2002) Molecular basis of quantitative factor $\mathrm{V}$ deficiency associated with factor $\mathrm{V}$ R2 haplotype. Blood. 100:2515-21.

16. Mikkola H, Muszbek L, Haramura G, Hamalainen E, Jalanko A, Palotie A. (1997) Molecular mechanisms of mutations in factor XIII A-subunit deficiency: in vitro expression in COS-cells demonstrates intracellular degradation of the mutant proteins. Thromb. Haemost. 77:1068-72.

17. von Heijne G. (1990) The signal peptide. J. Membr Biol. 115:195-201.

18. von Heijne G. (1985) Signal sequences: the limits for variation. J. Mol. Biol. 184:99-105.

19. Emanuelsson $\mathrm{O}$, Nielsen $\mathrm{H}$, Brunak $\mathrm{S}$, von Heijne G. (2000) Predicting subcellular localization of proteins based on their N-terminal amino acid sequence. J. Mol. Biol. 300:1005-16.

20. Yang F, Moss LG, Phillips GN Jr. (1996) The molecular structure of green fluorescent protein. $\mathrm{Na}$ ture Biotech. 14:1246-51.

21. Rapaport SI, Rao RVM. (1995) The tissue factor pathway: how it has become a "prima ballerina." Thromb. Haemost. 74:7-17.

22. Cooper DN, Millar DS, Wacey A, Banner DW, Tuddenham EGD. (1997) Inherited factor VII deficiency: molecular genetics and pathophysiology. Thromb. Haemost. 78:151-60.

23. Ozawa T, Takikawa Y, Niiya K, Ejiri N, Suzuki K, Sato S, Sakuragawa N. (1998) Factor VII Morioka (FVII L-26P): a homozygous missense mutation in the signal sequence identified in a patient with factor VII deficiency. Br. J. Haematol. 101:47-9.

24. Wulff K, Herrmann FH. (2000) Twenty two novel mutations of the factor VII gene in factor VII deficiency. Hum. Mutat. 15:489-96.

25. Pinotti M, Toso R, Redaelli R, Berrettini M, Marchetti G, Bernardi F. (1998) Molecular mechanisms of FVII deficiency: expression of mutations clustered in the IVS7 donor splice site of factor VII gene. Blood. 92:1646-51.

26. Chiesa A, Rapizzi E, Tosello V, Pinton P, de Virgilio M, Fogarty KE, Rizzuto R. (2001) Recombinant aequorin and green fluorescent protein as valuable tools in the study of cell signaling. Biochem. J. 355:1-12.

27. Toso R, Pinotti M, High KA, Pollak ES, Bernardi F. (2002) A frequent human coagulation Factor VII mutation (A294V, c152) in loop 140s affects the interaction with activators, tissue factor and substrates. Biochem. J. 363:411-6.

28. Pinotti M et al. (2002) Residual factor VII activity and different hemorrhagic phenotypes in $\mathrm{CRM}(+)$ factor VII deficiencies (Gly331Ser and Gly283Ser). Blood. 99:1495-7.
29. Kaufman RJ. (1998) Post-translational modifications required for coagulation factor secretion and function. Thromb. Haemost. 79:1068-79.

30. Katsumi A et al. (1998) The carboxyl-terminal region of protein $\mathrm{C}$ is essential for its secretion. Blood. 91:3784-91.

31. Greer J. (1990) Comparative modeling methods: application to the family of the mammalian serine proteases. Proteins 7:317-34. 